\title{
On the Magnetic Field Configuration of the Magnetosheath
}

\author{
Steven Michael Petrinec* \\ Lockheed Martin Advanced Technology Center, Palo Alto, California, USA
}

Received 16 April 2012, accepted 17 October 2012

\begin{abstract}
The magnetosheath is the region where the shocked solar wind interacts directly with the magnetosphere. Here we examine the magnetic field throughout the equatorial magnetosheath as a function of the solar wind IMF direction using observational data sets (Geotail and Wind) spanning 9.5 years. In general, the synoptic maps of the magnetosheath magnetic field are in agreement with the predictions of Rankine-Hugoniot conditions at the bow shock and with global numerical models. Qualitative comparisons with recent analytic magnetosheath magnetic field models are also performed and show that the compression of the magnetosheath magnetic field between the two boundaries (bow shock and magnetopause) used in such models is greater than that which is observed.
\end{abstract}

Key words: Magnetosheath, Bow shock, MHD discontinuities, Magnetopause

Citation: Petrinec, S. M., 2013: On the magnetic field configuration of the magnetosheath. Terr. Atmos. Ocean. Sci., 24, 265-272, doi: 10.3319/TAO. 2012.10.17.02(SEC)

\section{INTRODUCTION}

The interaction of the (shocked) solar wind and the Earth's magnetic field has been the subject of considerable study since the 1960s (Spreiter et al. 1966; Fairfield 1967), after it was established through spacecraft observations that solar wind plasma is a consistent feature of interplanetary space (Gringauz et al. 1960; Neugebauer and Snyder 1962, 1966).

The solar wind has long been understood to behave as a collisionless magnetohydrodynamic (MHD) gas over large spatial and lengthy time scales. The bow shock has been recognized as a standing fast mode shock wave between the supermagnetosonic solar wind and magnetospheric obstacle. Thus, the change in plasma parameters and magnetic field across the bow shock follow Rankine-Hugoniot jump conditions. It has also been long understood that the interaction between the shocked solar wind and the magnetosphere is characterized as a tangential discontinuity. At this boundary (the magnetopause), the shocked solar wind magnetic field is draped tangentially to the magnetopause surface (ignoring for the moment non-ideal MHD processes such as magnetic reconnection). The region between the bow shock and

\footnotetext{
* Corresponding author

E-mail: steven.m.petrinec@lmco.com
}

magnetopause is the magnetosheath where the shocked solar wind is slowed and diverted around the magnetopause.

The magnetic field of the magnetosheath is therefore treated to zeroth order as a boundary value problem, such that the jump conditions across the bow shock are well-understood, and the boundary condition $B_{n}=0$ at the magnetopause is understood. However, the region between these two boundaries is not at all well-understood. This is due in part to the difficulty in determining the locations and shapes of these boundaries (e.g., the magnetopause is not a rigid obstacle, but is self-consistent in pressure balance with the shocked solar wind, and the bow shock is located in a selfconsistent manner in relation to the magnetopause boundary), and the effects of other plasma processes. Although it is understood that the magnetosheath magnetic field is tangent to the magnetopause, its orientation within the tangential plane is not analytically understood. The orientation at the magnetopause is extremely important because the relation between the magnetosheath and magnetospheric magnetic fields across the magnetopause is believed to determine where (and perhaps at what rate) magnetic reconnection occurs.

Some recent statistical studies of the magnetosheath magnetic field have focused on the far downstream magnetosheath region (at $X \sim-30 \mathrm{R}_{\mathrm{E}}$ using IMP-8 observations, 
Kaymaz 1998), the low-latitude dawnside flank region adjacent to the magnetopause (using Equator-S observations, Dunlop et al. 1999), and the draping configuration near the magnetopause, Coleman (2005). In this study, we use the in situ magnetic field observations from Geotail spacecraft to produce synoptic maps of the equatorial magnetosheath region field intensity and orientation within the plane for various interplanetary magnetic field (IMF) orientations. Qualitative comparisons with analytic models are also performed.

\section{EMPIRICAL RESULTS}

The observations used in this study of the magnetosheath magnetic field are from the Geotail spacecraft (Kokubun et al. 1994). The Geotail orbit is at low inclination; thus, this study is focused on the magnetosheath in the equatorial region. The magnetosheath intervals have been identified by direct observation by the author, high-school students and teachers hired for the summer through the Industry Initiatives for Science and Math Education program (IISME). Bow shock crossings are readily identified as sudden changes in the ion moments and magnetic field intensity which are not present in the solar wind in accordance with that expected from Rankine-Hugoniot relations. The magnetopause crossings are best identified as a change in the magnetic field clock angle from that which varies in close agreement with the IMF clock angle to that which is steady and independent of the IMF clock angle. The magnetosheath intervals span the period from April 1996 through October 2005. This extended time span resulted in 6175 separate magnetosheath intervals, though it is noted that many of these intervals are quite brief as a result of boundary motions. There are three basic categories of magnetosheath intervals: (1) magnetopause to bow shock or bow shock to magnetopause (a sample crossing is shown in Fig. 1); (2) bow shock to bow shock (Fig. 2); and (3) magnetopause to magnetopause (Fig. 3).

The magnetosheath intervals were then matched with convected solar wind observations from the Wind spacecraft (Lepping et al. 1995; Ogilvie et al. 1995). Solar wind and magnetosheath parameters have been averaged down to a 5-minute resolution. The choice of this time resolution was based on several factors. First, the Wind plasma moment parameters at higher resolution (several tens of seconds) are not provided at uniform cadence. Second, it is believed that a higher time resolution does not improve the analyses, since the neighboring samples in time are less likely to be statistically independent. Last, the convection time from the solar wind monitor to the magnetosheath-sampling spacecraft is not known to be better than a few minutes especially since the plasma velocity is slowed after crossing the bow shock.

The convection time was taken as $\Delta \mathrm{x} / \mathrm{V}_{\mathrm{sw}}$ up to the bow shock, with an additional $\Delta \mathrm{t}^{\prime}=\Delta \mathrm{x}^{\prime} / \mathrm{V}_{\mathrm{sw}} / 3$ for those locations within the magnetosheath. The aberration angle of the solar wind at each instance was used to adjust the magnetosheath locations into an aberrated Geocentric Solar Ecliptic (GSE) coordinate system.

The magnetosheath intervals occur during various solar wind conditions; therefore, the boundaries during these

(a)

(b)

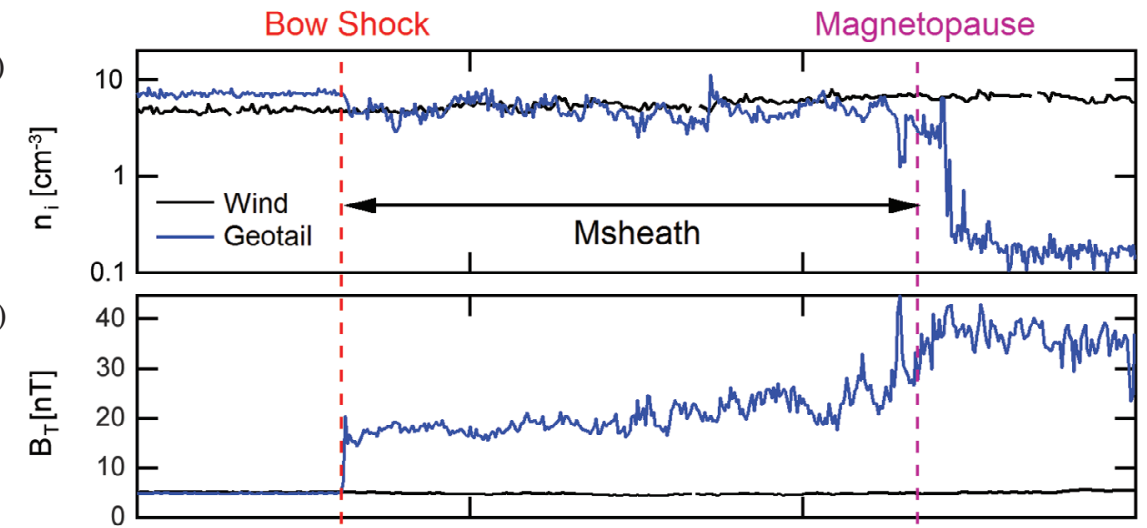

(c)

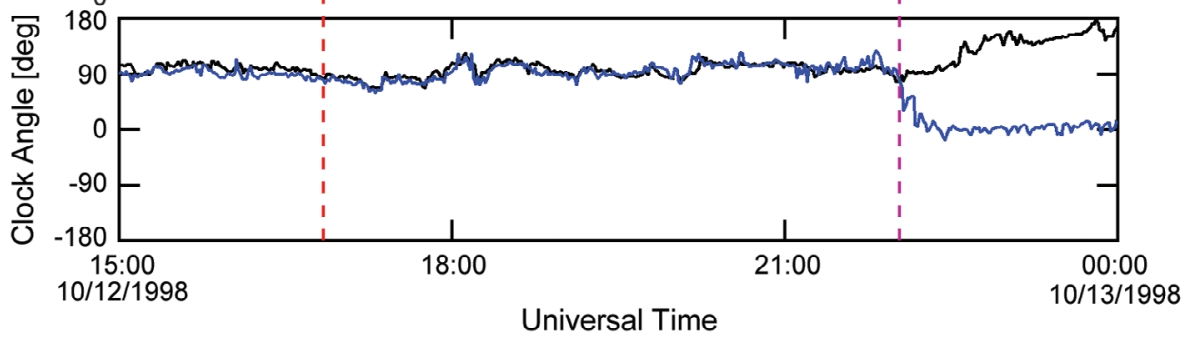

Fig. 1. An example of a magnetosheath interval, as the Geotail spacecraft crosses from the solar wind to the magnetosphere (blue traces). (a) Ion density. (b) Magnetic field intensity. (c) IMF clock angle. The convected Wind observations (black traces) are included for solar wind context. 
times are crossed at various distances. To produce synoptic maps of the magnetic field, the next step was to normalize the magnetosheath passes to common inner and outer boundaries by using empirical models as parameterized by the solar wind condition. The models used in this study are the Farris and Russell (1994) model for the bow shock and the Shue et al. (1998) magnetopause model for a solar wind dynamic pressure of $2 \mathrm{nPa}$ and zero IMF $\mathrm{B}_{\mathrm{z}}$. The normalization process scales many of the magnetosheath passes such that they begin and end at the empirical model boundaries. However, due to boundary oscillations and other dynamics and uncertainties, many of the normalized passes do not 'fit'

(a)

(b)

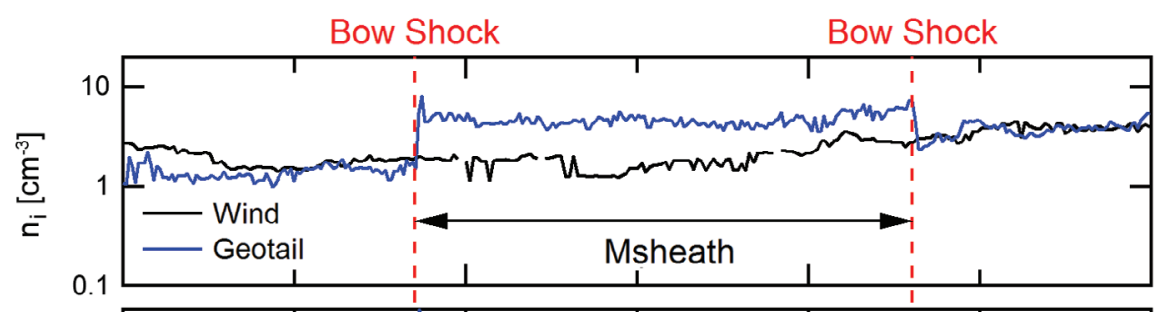

(c)
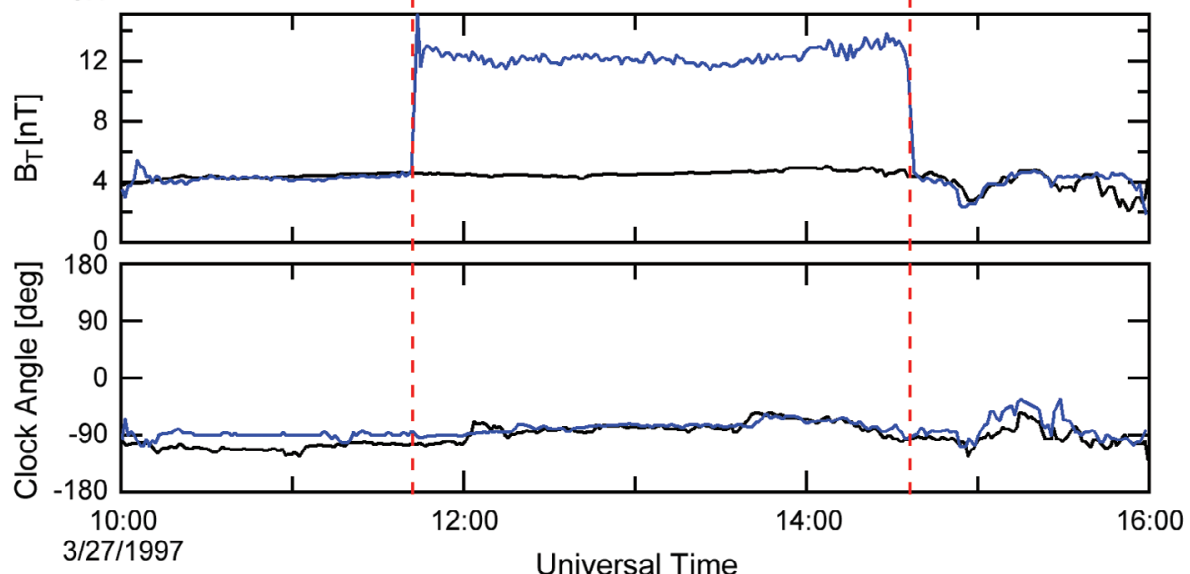

Fig. 2. A sample Geotail magnetosheath interval, bounded on both sides by the bow shock. The format is the same as Fig. 1 .

(a)

(b)

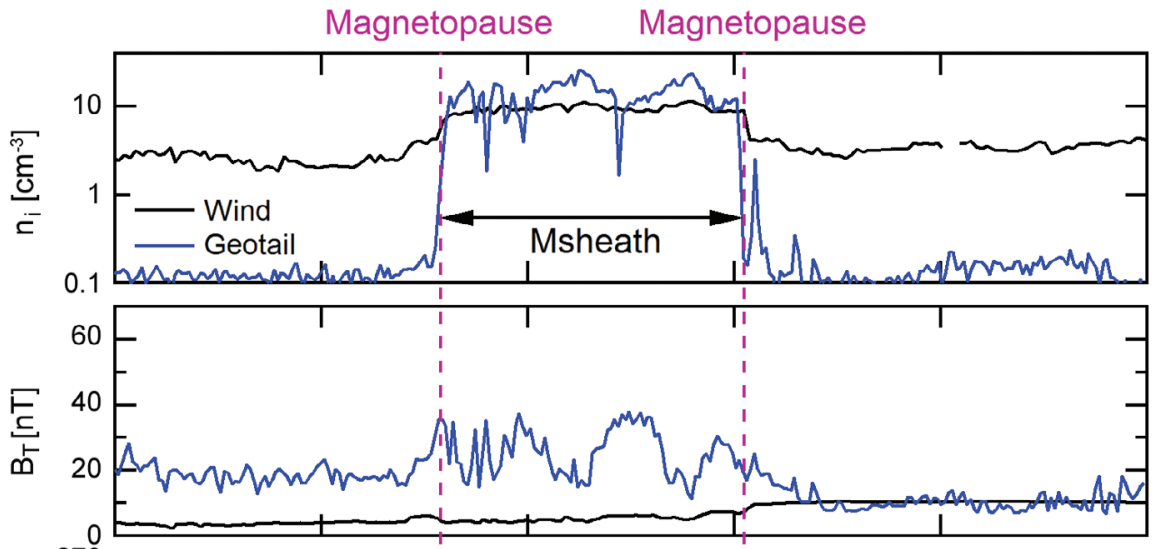

(c)

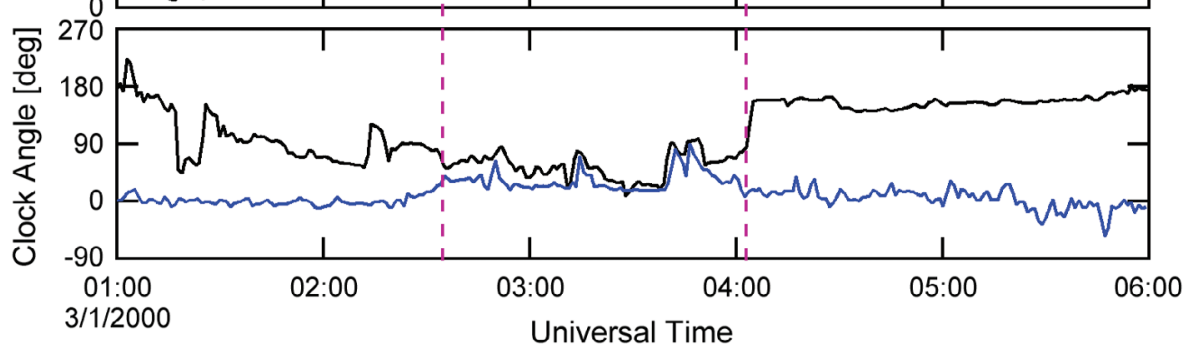

Fig. 3. A sample Geotail magnetosheath interval bounded on both sides by the magnetopause. The format is the same as Fig. 1 . 
between the boundaries. Therefore, a second normalization process has been used to force the ends of each pass to match the magnetosheath boundaries. Specifically, if the portion of a pass which has been identified as 'magnetosheath' has end points which do not match the model boundaries (after normalizing by the solar wind parameters), then the spatial locations relating to the pass are linearly scaled (typically by a few percent at most) such that the endpoints do match the boundaries.

The 5-minute samples of the magnetosheath magnetic field were then filtered by the orientation of the IMF and collected into Cartesian bins according to the aberrated and normalized location of the spacecraft. For those bins within which there were at least 6 samples; the median magnetic field strength (normalized by the IMF intensity) and the median magnetic field orientation are determined. Medians values are used so that any dynamics or errors in the estimated solar wind convection time which produce outliers do not significantly skew the determination of the 'typical' magnetic field.

In Fig. 4, synoptic maps of the aberrated GSE equatorial plane are created for the cases when the IMF is within $20^{\circ}$ of the normal to the solar wind flow direction and for which the absolute value of the $\mathrm{z}$-component of the IMF is small compared with the root-mean-square of the $\mathrm{x}$ - and $\mathrm{y}$ - components $\left[\left|\mathrm{B}_{z}\right|<\left(\mathrm{B}_{\mathrm{x}}^{2}+\mathrm{B}_{\mathrm{y}}^{2}\right)^{1 / 2} / 2\right]$. Figure $4 \mathrm{a}$ shows a contour plot produced from the median values of the intensity of the magnetosheath magnetic field normalized by the intensity of the IMF $\left(B_{T} / B_{T-I M F}\right)$, at $0.5 \times 0.5 \mathrm{R}_{\mathrm{E}}$ spatial resolution. The magnetic field intensity ratio near the subsolar bow shock is greater than three which is expected from the Rankine-Hugoniot relations. Figure $4 \mathrm{~b}$ shows the median magnetic field vector orientation within this plane in $1 \times$ $1 \mathrm{R}_{\mathrm{E}}$ bins (blue line segments). The resulting vector directions are also as expected; the vector field smoothly varies throughout the magnetosheath, and is not significantly different between dawn and dusk. The contour levels of the intensity ratio are also similar between dawn and dusk. To present this more explicitly, the median intensity as a function of $X_{\mathrm{aGSE}}$ along the dawn and dusk directions are shown in Fig. 5. These values are calculated in 15 angular bins from an origin located just inside of the subsolar magnetopause $\left(9 \mathrm{R}_{\mathrm{E}}\right)$. The $X_{\mathrm{aGSE}}$ ranges (horizontal bars) in Fig. 5 are determined from the intersection of the angular bins with the magnetosheath region. Markers are placed at the center of the $X_{\mathrm{aGSE}}$ range. The vertical error bars display the first and
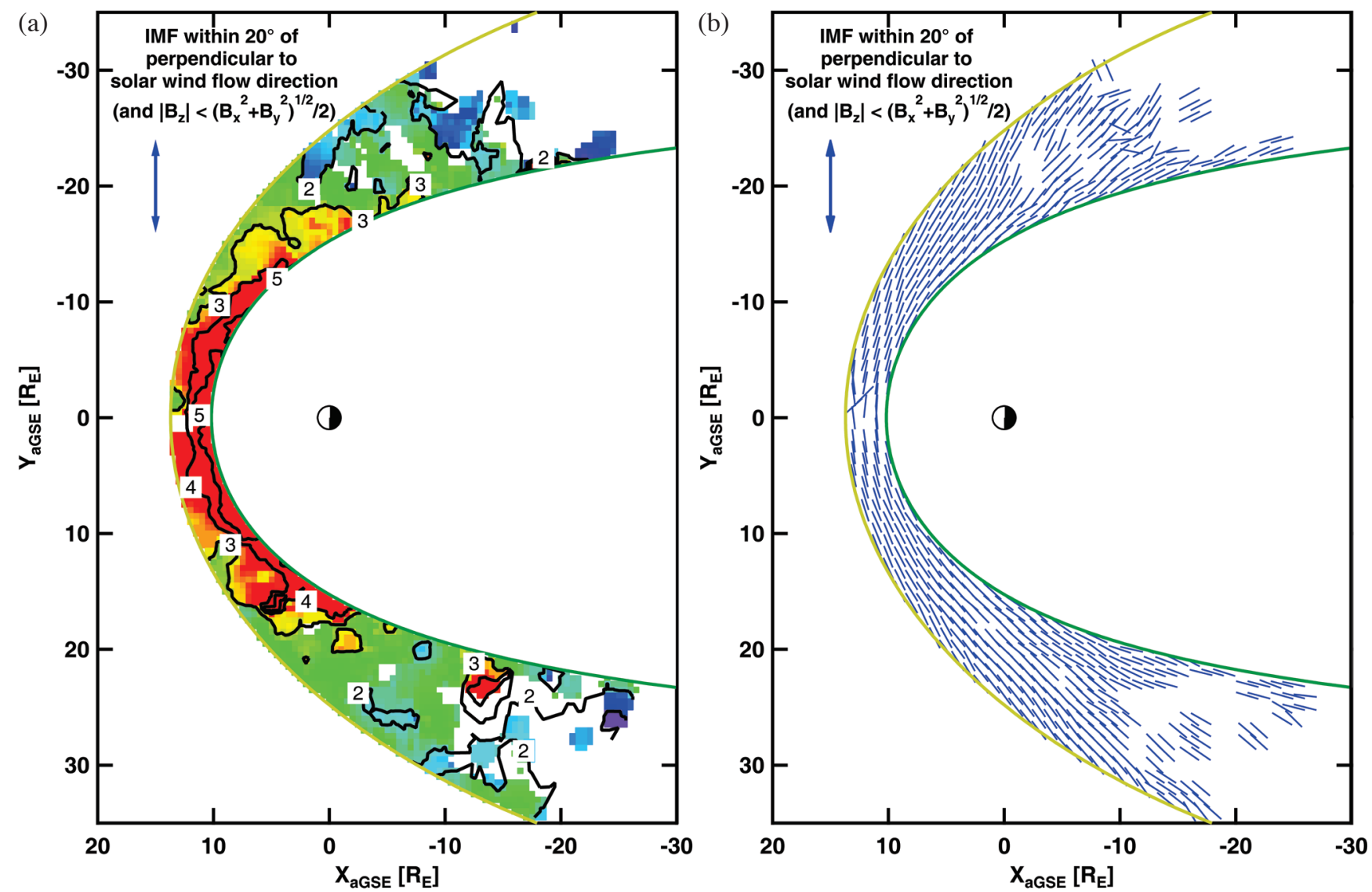

Fig. 4. All Geotail magnetic field observations of the equatorial magnetosheath, when the IMF is within $20^{\circ}$ of perpendicular to the solar wind flow direction and for small $\mid$ IMF $B_{z} \mid$. Contour plot of the normalized magnetic field intensity (a) and orientation of the magnetosheath magnetic field (b). 
third quartiles of the magnetic field intensity ratio. As can be seen, the dawn and dusk magnetosheath field ratios are approximately equal, as expected for IMF conditions which have large $\mathrm{B}_{\mathrm{y}}$ - component compared to either the $X$ - or $Z$ components.
Figure 6 is similar to Fig. 4, but is filtered to those samples for which the IMF lies within $10^{\circ}$ of the nominal Parker-Spiral angle (i.e., $\sim-45^{\circ} / 135^{\circ}$ from the Sun-Earth line). In this case, the magnetic field intensity ratio contours (left) are larger in the duskside than in the dawnside dayside

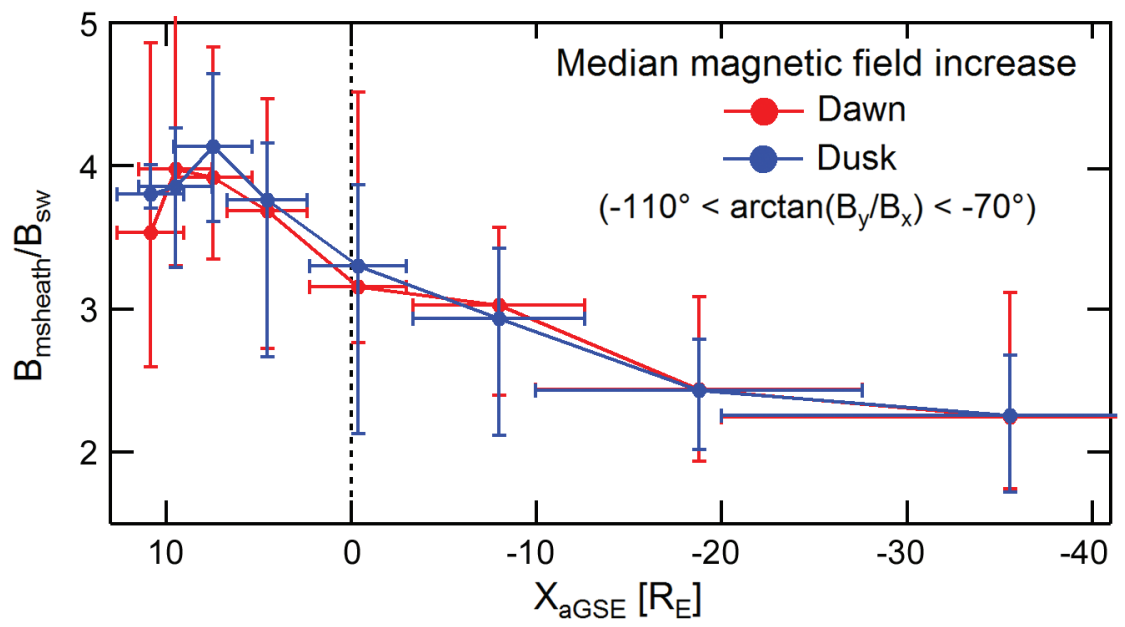

Fig. 5. Median magnetic field increase in the magnetosheath along dawn and along dusk, as a function of $X$-distance. Vertical error bars denote the first and third quartile values.
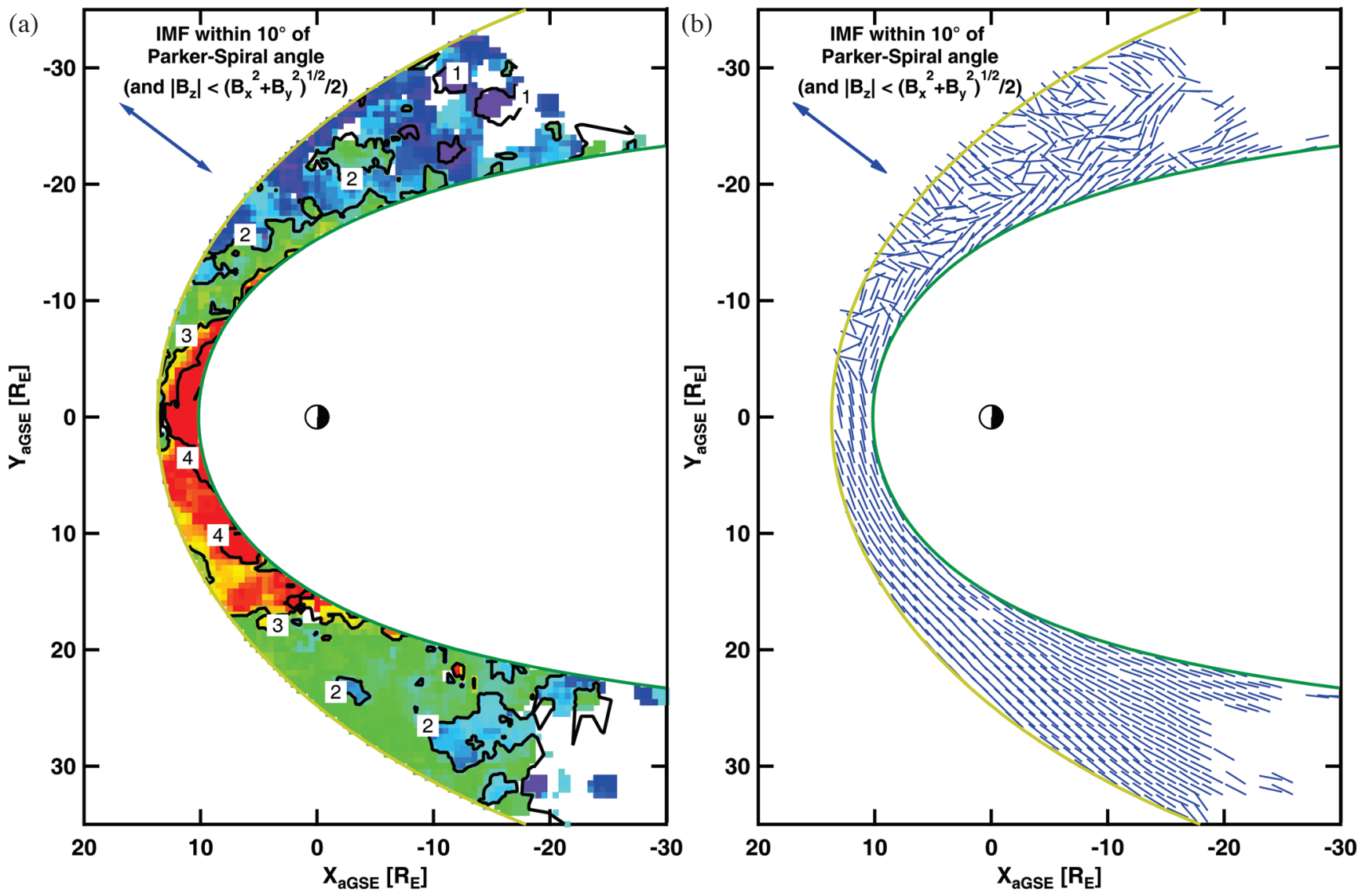

Fig. 6. The same format as Fig. 4, but for IMF intervals which lie close to the Parker-spiral angle direction. 
magnetosheath. Near the dayside bow shock surface, the variation of the magnetic field ratio is again as expected from Rankine-Hugoniot relations (Petrinec and Russell 1997).

The right side of Fig. 6 again shows the median orientation directions of the equatorial components of the magnetosheath magnetic field. The chaotic nature of the magnetic field directions in the dawn side magnetosheath region is consistent with previous studies of the dynamics and shock reformation processes associated with the quasi-parallel bow shock. On the dusk side magnetosheath, the orientation vectors are much more ordered.

In Fig. 7 , the IMF orientation atan $\left(B_{y} / B_{x}\right)$ is limited to angles within the equatorial plane between $-10^{\circ}$ and $-30^{\circ}$ from the radial flow direction, towards the direction of the Parker-Spiral direction (at $\sim-45^{\circ} / 135^{\circ}$ ). Similar to Fig. 6, the magnetic field intensity ratio is larger in the dusk than the dawn dayside magnetosheath region; as expected, the median magnetic field vectors are better aligned in the dusk than the dawn magnetosheath.

Figure 8 shows the median magnetosheath magnetic field deviation angle from the $\mathrm{X}$-axis along the dusk magnetosheath, for the three IMF cases shown in Figs. 4, 6, and 7. In the dayside region, the deviation angle is closest to $90^{\circ}$, and slowly decreases with increasing distance downtail as the magnetic field drapes around the magnetopause obstacle. The x-ranges in Fig. 8 are the same as is used in Fig. 5. The vertical error bars represent the first and third quartiles of the field deviation angle within each bin. It is seen that the median deviation angle varies smoothly with distance down the dusk magnetosheath flank. The different IMF conditions cause the deviation (draping) angle to vary at different rates along the duskside magnetosheath.

Last, a quick comparison of the magnetic field intensity ratios is made between the observations and recent analytic models. Two analytic magnetic field models describing the magnetosheath have been put forth by Kobel and Flückiger (1994) and by Romashets et al. (2008). The Kobel and Flückiger model determines a magnetic scalar potential within a region bounded by two paraboloids representing the magnetopause and bow shock. The scalar potential is expressed in terms of the IMF, and the magnetosheath magnetic field is then determined from the scalar potential. The Romashets et al. (2008) model uses the same boundaries, but solves for the magnetic vector potential, thus allowing for a non-zero distributed current density within the magnetosheath.
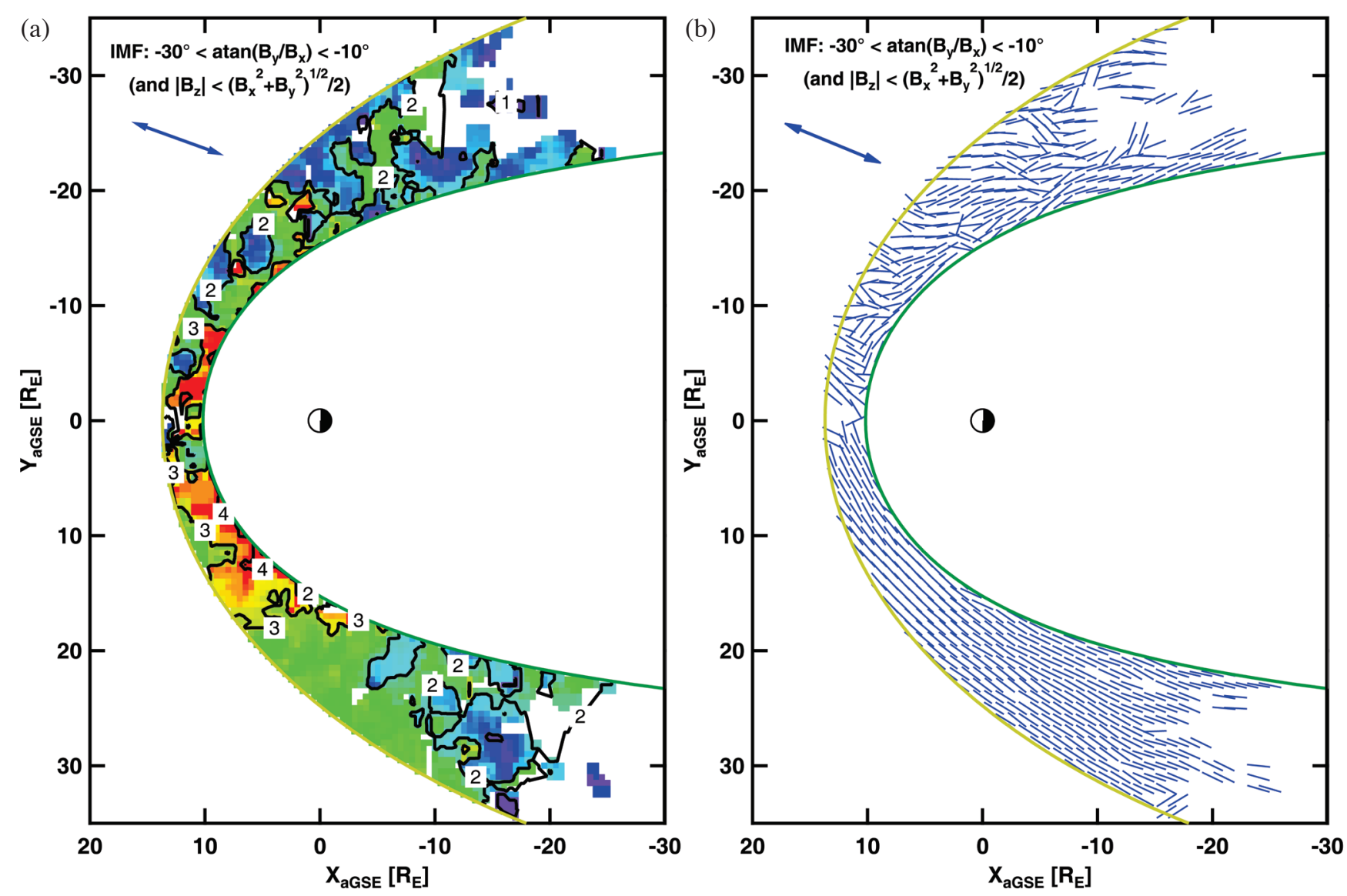

Fig. 7. The same format as Fig. 4, but for IMF intervals which lie between the solar wind flow direction and the Parker-spiral angle direction. 
In Fig. 9, we plot the magnetic intensity ratio within the magnetosheath region of both analytic models for a Parker-spiral IMF configuration. The resulting maps from the two models are very similar, with higher field strength in the subsolar region and along the dusk flank than in the dawn flank region. A qualitative comparison between these results and those shown in Fig. 6a illustrates that there are considerable differences between the observations and the analytic models. In particular, the magnetic field intensity ratios on the dusk side flanks are significantly higher in the analytic models than in the observations, and are higher than expected from the Rankine-Hugoniot relations across the dusk side bow shock. It is suggested that the reason for the discrepancy is that the use of paraboloid shapes for both boundaries in the analytic models allows too little room for the magnetic flux tubes to pass around the obstacle in the

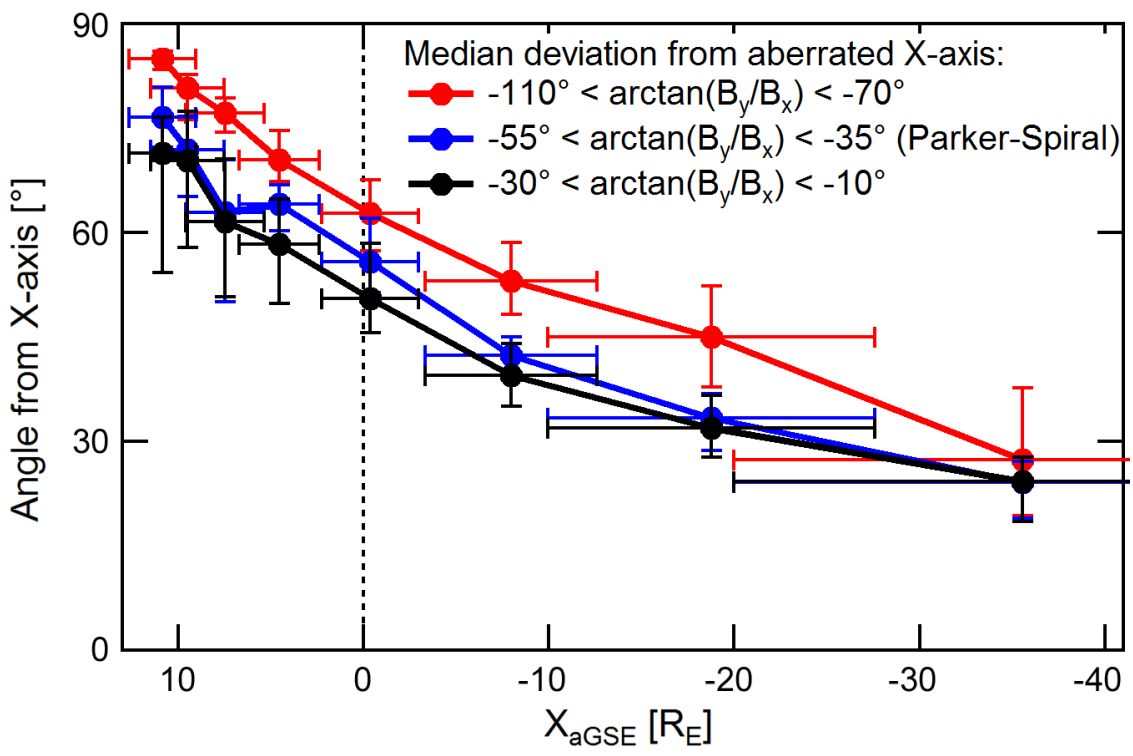

Fig. 8. The median angle of magnetic field draping (with respect to the $X$-axis) along the dusk magnetosheath, as a function of distance along the $X$-axis. The three traces represent the IMF cases shown in Figs. 4 (red), 6 (blue), and 7 (black). Horizontal bars represent the range of $X_{\mathrm{aGSE}}$ in the magnetosheath bin. Vertical error bars represent the first and third quartiles.
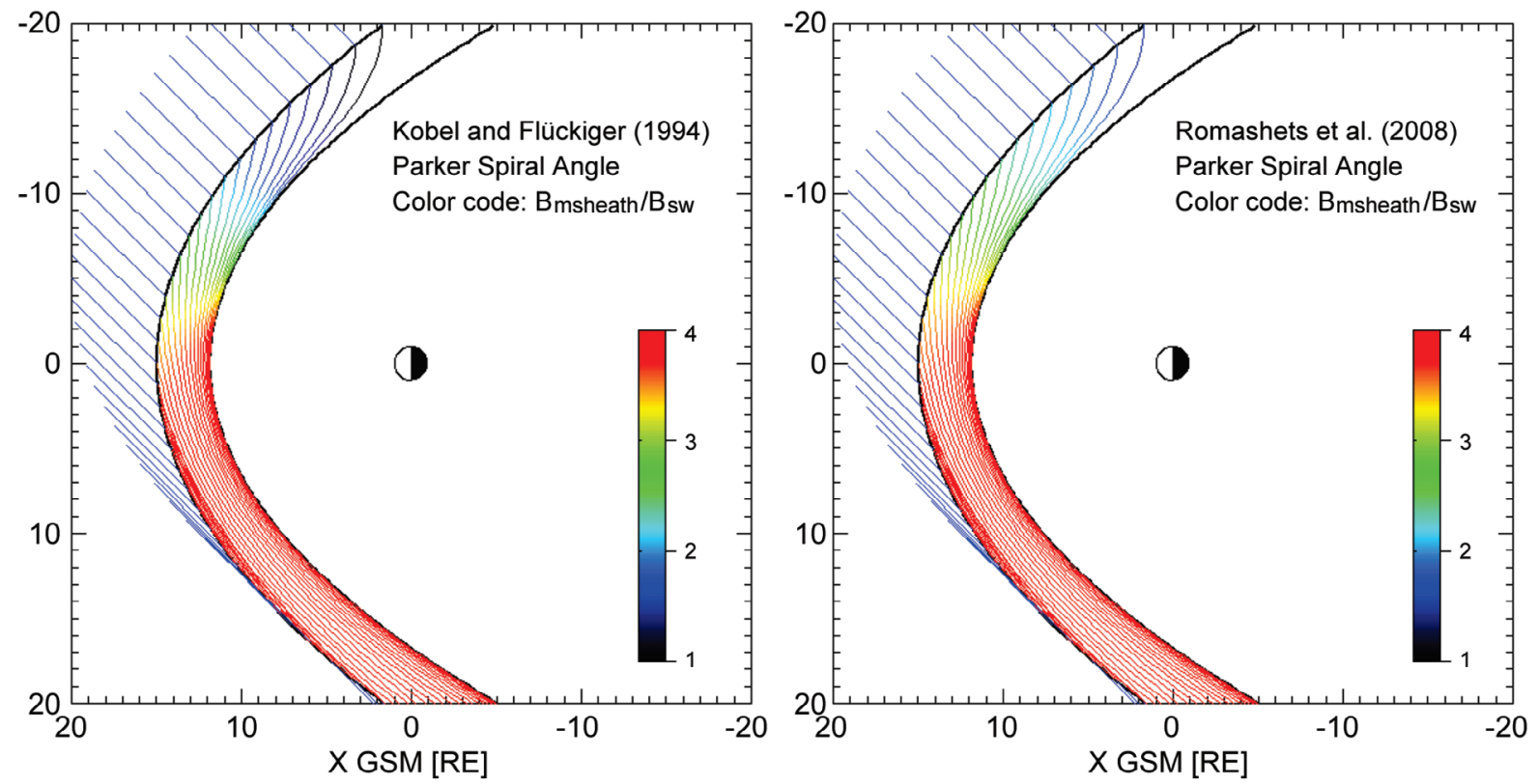

Fig. 9. Magnetic field intensity ratio maps for two analytic magnetic field models, for the Parker-spiral angle IMF configuration. 
same way as in nature, and that different boundary shapes need to be considered.

\section{CONCLUSIONS}

The large-scale structure of the magnetosheath magnetic field intensity and orientation as observed by the Geotail spacecraft is in accordance with the general knowledge of expected behavior. The jump in the magnetic field intensity across the bow shock is in good agreement with Rankine-Hugoniot relations. Also, the magnetic field in the region downstream of the quasi-parallel bow shock is more disordered than downstream of the quasi-perpendicular bow shock. Finally, it is recognized from a qualitative comparison of the observations with analytic models that more work needs to be done to improve the analytic magnetosheath models. Specifically, it is believed that the use of boundary shapes which allow for greater magnetosheath thickness in the flank regions will result in more favorable comparisons of the flank magnetosheath magnetic field intensity between analytic models and observations.

Acknowledgements The author wishes to thank the Geotail and Wind instrument and science teams for providing the observations to the public via CDAWeb. In addition, the author thanks Elle Allen, Katy Kuei, Marcos Pedreiro, and Janine Stovall for their efforts and contributions in identifying geophysical boundaries in the Geotail data sets. This work has been supported by NASA grants NNG05GE15G and NNX11AI35G.

\section{REFERENCES}

Coleman, I. J., 2005: A multi-spacecraft survey of magnetic field line draping in the dayside magnetosheath. Ann. Geophys., 23, 885-900, doi: 10.5194/angeo-23885-2005. [Link]

Dunlop, M. W., A. Balogh, W. Baumjohann, G. Haerendel, K.-H. Fornacon, and E. Georgescu, 1999: Dawnside magnetopause observed by the Equator-S Magnetic Field Experiment: Identification and survey of crossings.J.Geophys.Res., 104, 17491-17497, doi: 10.1029/ 1999JA900214. [Link]

Fairfield, D. H., 1967: The ordered magnetic field of the magnetosheath. J. Geophys. Res., 72, 5865-5877, doi: 10.1029/JZ072i023p05865. [Link]

Farris, M. H. and C. T. Russell, 1994: Determining the standoff distance of the bow shock: Mach number dependence and use of models. J. Geophys. Res., 99, 17681-17689, doi: 10.1029/94JA01020. [Link]

Gringauz, K. I., V. V. Bezrukikh, V. D. Ozerov, and R. Ye. Rybchinsky, 1960: A study of interplanetary ionized gas, energetic electrons, and corpuscular solar emission, using three-electrode charged-particle traps set up on the second Soviet cosmic rocket Luna 2. Dokl. Akad. Nauk USSR, 131, 1301-1304.

Kaymaz, Z., 1998: IMP 8 magnetosheath field comparisons with models. Ann. Geophys., 16, 376-387, doi: 10.1007/s00585-998-0376-3. [Link]

Kobel, E. and E. O. Flückiger, 1994: A model of the steady state magnetic field in the magnetosheath. J. Geophys. Res., 99, 23617-23622, doi: 10.1029/94JA01778. [Link]

Kokubun, S., T. Yamamoto, M. H. Acuña, K. Hayashi, K. Shiokawa, and H. Kawano, 1994: The GEOTAIL magnetic field experiment. J. Geomagn. Geoelectr., 46, 7-21.

Lepping, R.P., M. H. Acũna, L. F. Burlaga, W. M. Farrell, J. A. Slavin, K. H. Schatten, F. Mariani, N. F. Ness, F. M. Neubauer, Y. C. Whang, J. B. Byrnes, R. S. Kennon, P. V. Panetta, J. Scheifele, E. M. Worley, 1995: The Wind magnetic field investigation. Space Sci.Rev., 71, 207-229, doi: 10.1007/BF00751330. [Link]

Neugebauer, M. and C. W. Snyder, 1962: The mission of Mariner 2: Preliminary observations, solar plasma experiment. Science, 138, 1095-1097.

Neugebauer, M. and C. W. Snyder, 1966: Mariner 2 observations of the solar wind: 1 . Average properties. $J$. Geophys. Res., 71, 4469-4484, doi: 10.1029/JZ071i01 9p04469. [Link]

Ogilvie, K. W., D. J. Chornay, R. J. Fritzenreiter, F. Hunsaker, J. Keller, J. Lobell, G. Miller, J. D. Scudder, E. C. Sittler Jr., R. B. Torbert, D. Bodet, G. Needell, A. J. Lazarus, J. T. Steinberg, J. H. Tappan, A. Mavretic, E. Gergin, 1995: SWE, A comprehensive plasma instrument for the Wind spacecraft. Space Sci.Rev., 71, 5577, doi: 10.1007/BF00751326. [Link]

Petrinec, S. M. and C. T. Russell, 1997: Hydrodynamic and MHD equations across the bow shock and along the surfaces of planetary obstacles. Space Sci. Rev., 79, 757-791, 10.1023/A:1004938724300. [Link]

Romashets, E. P., S. Poedts, and M. Vandas, 2008: Modeling of the magnetic field in the magnetosheath region. J. Geophys. Res., 113, doi: 10.1029/2006JA0120 72. [Link]

Shue, J.-H., P. Song, C. T. Russell, J. T. Steinberg, J. K. Chao, G. Zastenker, O. L. Vaisberg, S. Kokubun, H. J. Singer, T. R. Detman, H. Kawano, 1998: Magnetopause location under extreme solar wind conditions. $J$. Geophys. Res., 103, 17691-17700, doi: 10.1029/98JA 01103. [Link]

Spreiter, J. R., A. L. Summers, and A. Y. Alksne, 1966: Hydromagnetic flow around the magnetosphere. Planet. Space Sci., 14, 223-250, doi: 10.1016/0032-0633 (66)90124-3. [Link] 